\title{
CONTROLO VETORIAL (FOC) DE UM MOTOR DE INDUÇÃO TRIFÁSICO APLICADO A
}

\section{UM VEÍCULO ELÉTRICO.}

\section{INTRODUÇÃO}

Os motores de indução (MI) com rotor em gaiola de esquilo estão muito disseminados nos atuais sistemas de variação de velocidade ("drives"). A sua simplicidade e robustez, aliadas a baixos preços (comparativamente com outras máquinas) e ampla gama de potências disponíveis, são as principais razões.

A evolução verificada nos domínios da eletrónica de potência e nos sistemas de controlo (em particular, o controlo digital), permitiram aplicar os $\mathrm{MI}$ em sistemas de elevado desempenho dinâmico (e.g., controlo de binário e/ou posição), substituindo os motores DC, cujas características dinâmicas e simplicidade de controlo os tornavam a primeira escolha em tais aplicações. Com efeito, as "drives" baseadas em $\mathrm{Ml}$ apresentam características dinâmicas em tudo semelhantes aos sistemas DC, incluindo a possibilidade de funcionamento nos quatro quadrantes do plano $\left(T, n_{r}\right)$. No entanto, a complexidade do conversores e, sobretudo, dos sistemas de controlo é muito mais elevada nos sistemas AC. Os sistemas baseados no controlo vetoria são os mais usuais nas "drives" baseadas nas máquinas AC convencionais (assíncronas e síncronas). Existem outras metodologias também usadas na indústria (e.g., controlo direto do binário - DTC), mas neste trabalho somente o controlo vetorial será abordado.

No domínio do controlo vectorial existem diversas variantes, sendo o método mais poderoso e utilizado o controlo por orientação de campo (Field Oriented Control - FOC). Desde finais da década de 60 do século passado, têm vindo a ser desenvolvidos vários métodos de controlo por orientação de campo [1]. Na sua essência, assentam numa filosofia semelhante aos sistemas DC: controlo independente do fluxo magnético e do binário desenvolvido. A sua implementação assenta na consideração de um referencial que gira com velocidade instantânea igual à do campo girantegirante (referencial síncrono), estando alinhado, em qualquer instante, com a posição desse mesmo campo .

O mais eficaz é sem dúvida o controlo por orientação do campo do rotor, sendo por isso o mais usual. No entanto, a implementação deste processo em AC é bastante mais complexa: para além dos valores das amplitudes é também necessário o controlo instantâneo da posição relativa dos fasores da expressão anterior, ou seja, é imprescindível o conhecimento, em cada instante, da posição espacial do fluxo do rotor em relação ao estator (i.e., referencial fixado ao estator).

\section{ROTOR FOC}

A implementação do controlo por orientação de campo rotórico assenta na conversão da máquina polifásica em análise (não necessariamente trifásica), num sistema bifásico equivalente (eixos ortogonais $d-q$ ), definido no referencial síncrono $\omega_{\psi r}$. A Figura 1 ilustra os conceitos associados ao controlo por orientação do campo do rotor - Rotor FOC (com base em [2]).

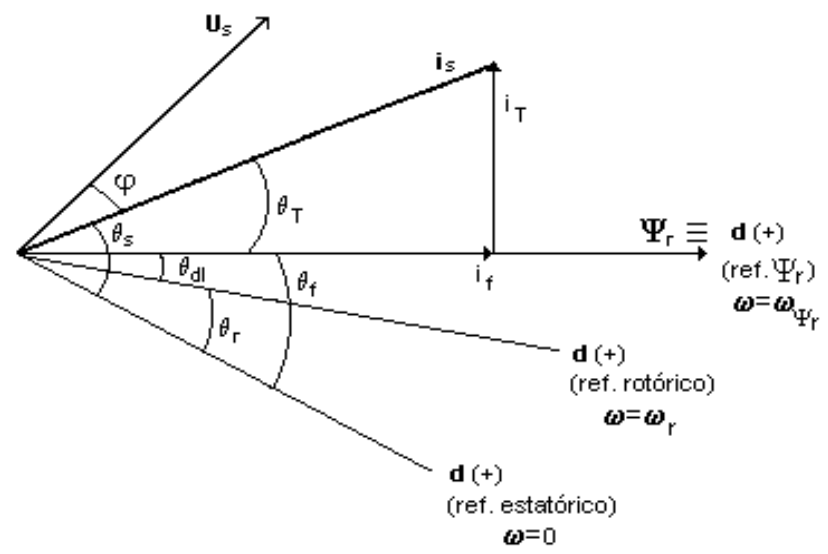

Figura 1. Controlo por orientação do campo do rotor em MI

O Rotor FOC assenta na definição das equações elétricas e magnéticas no referencial síncrono $\left(\omega_{\Psi \mathrm{r}}=2 \pi f / p\left(\mathrm{rads}^{-1}\right)\right)$, sendo a direção do fluxo do rotor alinhada, em cada instante, com o eixo $d$ desse referencial. As partes real e imaginária do fasor espacial corrente estatórica $\left(\mathbf{i}_{\mathbf{s}}\right)$ são, respetivamente, $\mathrm{i}_{\mathrm{f}}$ e $\mathrm{i}_{\mathrm{T}}$, pelo que:

\footnotetext{
${ }^{1}$ Poderá ser considerado qualquer um dos campos girantes presentes no motor: estator, entreferro ou rotor.
} 
$\mathrm{i}_{\mathrm{f}} \rightarrow$ alinhada com $\Psi \mathrm{r}$, regula o seu valor (eixo d);

$\mathrm{i}_{\mathrm{T}} \rightarrow$ desfasada de $\pi / 2$ rad. eléctricos em relação a $\mathrm{i}_{\mathrm{f}}$, controla o binário electromagnético desenvolvido (eixo q).

Em regime permanente tem-se:

$$
\begin{gathered}
\Psi_{r}=L_{m} i_{f} \\
T_{e l}=K_{T} \Psi_{r} i_{T}
\end{gathered}
$$

Em termos conceptuais, o controlo é implementado no referencial síncrono. No entanto, o controlador físico (hardware) actua ao nível do referencial do estator, isto é, sobre as tensões e correntes que alimentam o motor ( 3 fases $\left.\rightarrow\left(u_{a}, u_{b}, u_{c}\right),\left(i_{a}, i_{b}, i_{b}\right)\right)$. A determinação instantânea de $i_{f}$ e $i_{T}$ no referencial estático $(\omega=0)$ é fundamental. Uma vez que $\theta_{\mathrm{T}}=\operatorname{arctg}\left(\mathrm{i}_{\mathrm{T}} / \mathrm{i}_{\mathrm{f}}\right)$, a obtenção do valor instantâneo de $\theta_{\mathrm{f}}$ é o ponto central (simultaneamente, o mais exigente) na implementação do Rotor FOC.

\section{Rotor FOC - Método Indirecto}

Sendo esta a metodologia mais usualmente empregue, apresenta-se em seguida o modelo matemático do respectivo algoritmo de controlo. No essencial, $\theta_{f}$ é determinado através da medição de $\theta_{\mathrm{r}}$ e da estimação de $\theta_{\mathrm{dl}}$ (ver Figura 1).

Considerando a representação no sistema de eixos $d-q$, no referencial síncrono, as equações eléctricas do rotor de um MI com gaiola de esquilo são dadas por:

$$
\begin{aligned}
& 0=i_{r d} R_{r}+\frac{d \Psi_{r d}}{d t}-\left(\omega_{\Psi_{r}}-\omega_{r}\right) \Psi_{r q} \\
& 0=i_{r q} R_{r}+\frac{d \Psi_{r q}}{d t}+\left(\omega_{\Psi_{r}}-\omega_{r}\right) \Psi_{r d}
\end{aligned}
$$

Sendo: $\Psi_{\mathrm{rd}}=\Psi_{\mathrm{r}}$ e $\Psi_{\mathrm{rq}}=0$, as equações anteriores tomam a forma seguinte:

$$
0=i_{r d} R_{r}+\frac{d \Psi_{r}}{d t}
$$

$$
0=i_{r q} R_{r}+\left(\omega_{\Psi_{r}}-\omega_{r}\right) \Psi_{r}
$$

Por outro lado, as equações magnéticas do rotor, definidas no mesmo referencial, tomam a seguinte forma:

$$
\Psi_{r d}=\Psi_{r}=L_{r} i_{r d}+L_{m} i_{s d}
$$

$$
\Psi_{r q}=0=L_{r} i_{r q}+L_{m} i_{s q}
$$

Fixando: $i_{s d}=i_{f}$ e $i_{s q}=i_{T}$, vem que:

$$
\begin{aligned}
& i_{r d}=\frac{\Psi_{r}-L_{m} i_{f}}{L_{r}} \\
& i_{r q}=-\frac{L_{m}}{L_{r}} i_{T}
\end{aligned}
$$

Substituindo estas últimas expressões nas equações eléctricas do rotor, obtém-se:

$$
\begin{aligned}
& i_{f}=\frac{1}{L_{m}}\left(\Psi_{r}+T_{r o} \frac{d \Psi_{r}}{d t}\right) \\
& i_{T}=\frac{1}{L_{m}} T_{r 0}\left(\omega_{\Psi_{r}}-\omega_{r}\right) \Psi_{r}
\end{aligned}
$$

$\left[T_{r 0}\right.$ : constante de tempo do rotor $\mathrm{c} / \mathrm{o}$ estator em circuito aberto)]

Com base nas equações magnéticas do estator, também definidas no referencial $\omega_{\Psi r}$, o binário electromagnético instantâneo é dado por:

$$
T_{e l}=\frac{3}{2} p\left(\Psi_{s d} i_{s q}-\Psi_{s q} i_{s d}\right)=\frac{3}{2} p \frac{L_{m}}{L_{r}} \Psi_{r} i_{T}
$$

Assim, a velocidade associada ao deslizamento, $\omega_{\mathrm{d} l}$, expressa em função de $T_{\text {el }}$ e $\Psi_{\mathrm{r}}$ é dada por:

$$
\omega_{d l}=\omega_{\Psi r}-\omega_{r}=\frac{L_{m}}{T_{r 0}} \frac{i_{T}}{\Psi_{r}}=\frac{2 R_{r}}{3 p} \frac{T_{e l}}{\Psi_{r}^{2}}
$$


O campo girante do rotor gira com velocidade igual a $\omega_{\Psi r}$ relativamente ao referencial estatórico. Desta forma, considerando um instante $t_{0}$, tal que: $\theta_{f}\left(t_{0}\right)=\theta_{f 0} ; \theta_{r}\left(t_{0}\right)=\theta_{r 0} ; \theta_{d l}$ $\left(t_{0}\right)=\theta_{d 10}$, tem-se:

$$
\theta_{d l}(t)=\theta_{d l}\left(t_{0}\right)+\int_{t_{0}}^{t} \omega_{d i} d t
$$

Através da velocidade instantânea do rotor $\left(\omega_{\mathrm{r}}\right)$, obtém-se:

$$
\theta_{r}(t)=\theta_{r}\left(t_{0}\right)+\int_{t_{0}}^{t} \omega_{r} d t
$$

O valor de $\theta_{\mathrm{f}}$ é então obtido através de (ver Figura 1):

$$
\theta_{f}(t)=\theta_{d \mid}(t)+\theta_{r}(t)
$$

A conversão entre as mesmas grandezas definidas nos referenciais estático e síncrono é efetuada através da transformada de Park. Atendendo à ausência das componentes homopolares (dado que, usualmente, não existe condutor neutro nos $\mathrm{MI}$ ), esta transformada é dada por:

$\left[\begin{array}{l}i_{q s} \\ i_{d s}\end{array}\right]=\frac{2}{3}\left[\begin{array}{lll}\sin \theta_{e} & \sin \left(\theta_{e}-\frac{2}{3} \pi\right) & \sin \left(\theta_{e}+\frac{2}{3} \pi\right) \\ \cos \theta_{e} & \cos \left(\theta_{e}-\frac{2}{3} \pi\right) & \cos \left(\theta_{e}+\frac{2}{3} \pi\right)\end{array}\right]\left[\begin{array}{l}i_{s a} \\ i_{s b} \\ i_{s c}\end{array}\right]$

\section{SIMULAÇÃO}

O modelo de simulação utilizado baseia-se no conteúdo do bloco "Field Oriented Control Induction Motor Drive", integrado na "Electric Drives Library" do MATLAB/SIMULINK. Existem diversas limitações a considerar, sendo de referir:

- As perdas do conversor de potência não são consideradas;

- O modelo do motor não inclui as perdas no ferro;

- Não é possível efetuar frenagens regenerativas, somente dissipativas (i.e., sem recuperação de energia.
Em síntese, o modelo do sistema da cadeia de potência do veículo inclui somente a drive do motor e a transmissão mecânica. Deste modo, a tónica principal será dada ao desempenho do controlador, baseado no Rotor FOC (indireto). Em seguida, salientam-se algumas das condições mais relevantes a que o motor será submetido.

Na figura 2, está representado o modelo considerado da cadeia de potência do veículo.

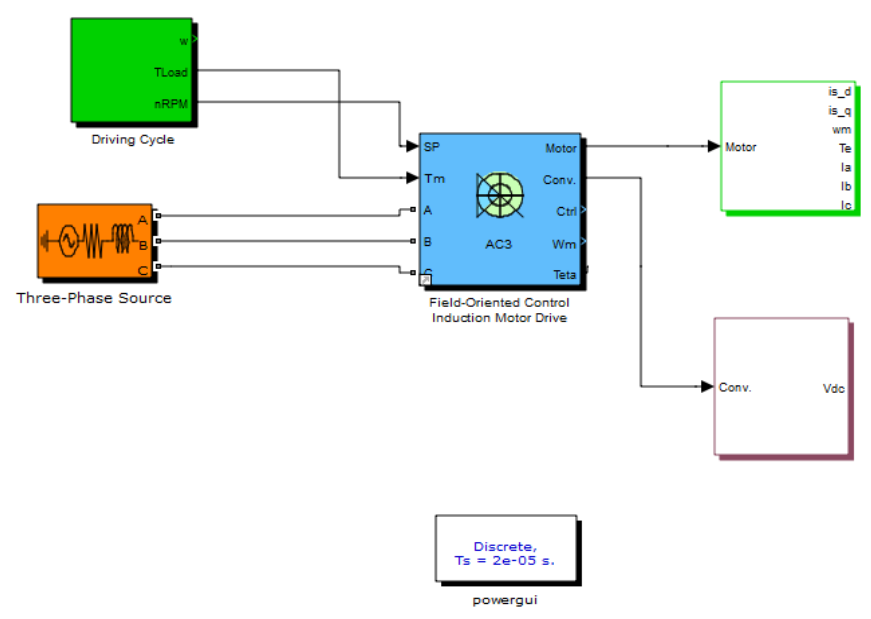

Figura 2. Modelo dinâmico do veiculo

Os principais blocos são o "Driving Cycle" (a verde, inclui o ciclo de condução pré-definido e os modelos do veículo e da transmissão) e o "Field Oriented Control Induction Motor Drive" (a azul, representa a drive do motor de indução).

\section{Ciclo de condução + Modelo do veículo (dinâmico e transmissão)}

Para a implementação dos modelos da transmissão mecânica e da dinâmica do veículo, recorreu-se à toolbox "QuasiStatic Simulation Toolbox" (QSS TB), desenvolvida por [3], em ambiente MATLAB/SIMULINK. Esta toolbox foi especificamente desenvolvida para a modelização de veículos híbridos e elétricos, com os seguintes elementos: ciclos de condução, modelo dinâmico do veículo, transmissão mecânica, motor de combustão interna e motor elétrico, baterias, super-condensadores e células de combustível (fuel-cells). Na figura 3 estão representados os elementos utilizados neste trabalho. 


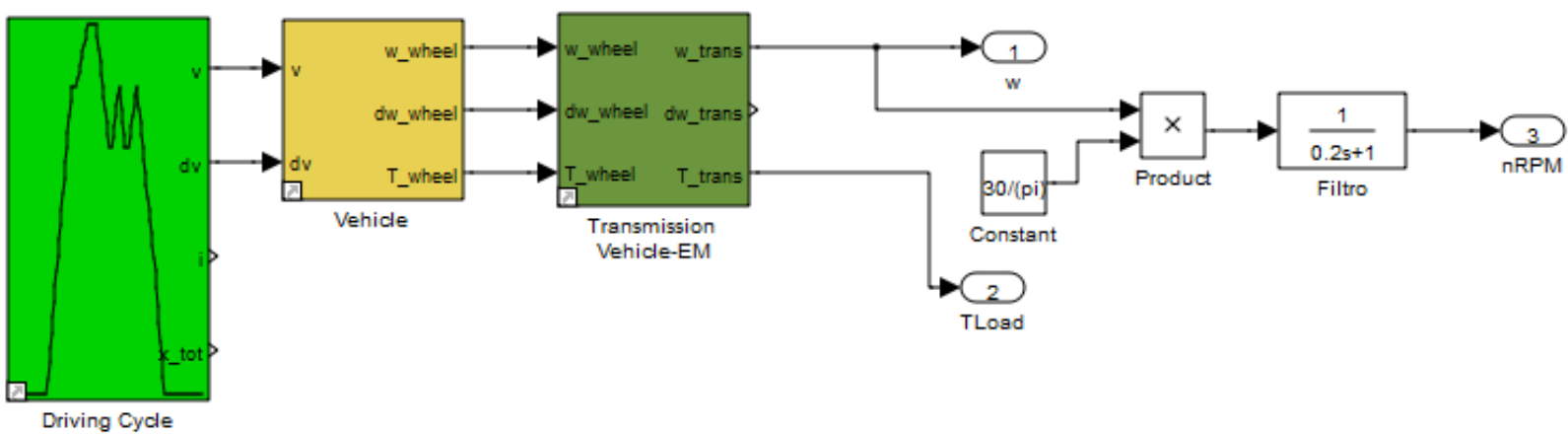

Figura 3. Ciclo de condução e modelo do veículo (dinâmica + transmissão

A potência instantânea pedida ao MI (Tload* $\left.\omega_{\mathrm{r}}\right)$ é calculada com base no ciclo de condução selecionado, no comportamento dinâmico do veículo (considera o atrito resultante do contacto roda-pavimento e a resistência aerodinâmica do ar, em deslocamentos planos) e num sistema de transmissão mecânico com uma razão constante.

A equação seguinte corresponde ao modelo dinâmico do veículo:

$$
M_{:} \frac{d v(t)}{d t}=F_{\delta}(t)-M_{:} g C_{s}-\frac{1}{2} \rho C_{m} A v(t)^{2}
$$

$M_{t} \quad$ Massa do veículo + massa equivalente dos elementos móveis;

v(t) Velocidade instantânea do veículo, (direção longitudinal);

$\mathrm{F}_{\mathrm{d}}(\mathrm{t})$ Força motora (instantânea) aplicada ao veículo, segundo a direção longitudinal;

g Aceleração gravítica [9.8 m`s-2];

$\mathrm{C}_{\mathrm{r}} ; \mathrm{C}_{\mathrm{w}}$ Coeficiente de atrito de rolamento; coeficiente de atrito aerodinâmico;

$\rho$; A Densidade do ar $\left[1.294 \mathrm{~kg} / \mathrm{m}^{3}\right]$; superfície frontal do veículo.

O 10 membro da equação anterior representa a força de inércia associada à aceleração do veículo, considerando também a variação da energia cinética acumulada nos componentes do veículo animados de movimento rotativo (Massa equivalente dos elementos móveis - Tabela 3).
Com efeito, tais variações da energia cinética são suportadas pelo motor.

Os parâmetros do veículo e do sistema de transmissão considerados estão indicados, respetivamente, nas Tabelas 1 e 2:

Tabela 1. Parâmetros do veículo

\begin{tabular}{lc}
\hline Massa (kg) & 350 \\
Massa equivalente dos elementos móveis (\%) & 1,5 \\
Secção Transversal $\left(\mathrm{m}^{2}\right)$ & 0,3 \\
Diâmetro da roda (m) & 0,3 \\
Coeficiente de atrito aerodinâmico & 0,008 \\
\hline $\begin{array}{l}\text { Coeficiente de atrito de rolamento } \\
\text { Tabela 2. Parâmetros da Transmissão Mecânica }\end{array}$ \\
\hline $\begin{array}{l}\text { Razão de transmissão } \\
\text { Rendimento (\%) }\end{array}$ \\
$\begin{array}{l}\text { Idling losses by friction (W) } \\
\text { Velocidade da roda acima da qual são } \\
\text { geradas perdas (rad/s) }\end{array}$ \\
\hline
\end{tabular}

A Tabela 3 contém os parâmetros do MI considerado.

Tabela 3. Parâmetros do motor de indução (7.5 kW; 400 V; 13 A; 50 Hz; 4 pólos; 1440 rpm)

\begin{tabular}{lc}
\hline$[\mathrm{Rs} ; \mathrm{Rr}](\Omega)$ & {$[0,7384 ; 0,7402]$} \\
{$[\mathrm{Ls} ; \mathrm{Lr}](\mathrm{H})$} & {$[127,14 ; 127,14]^{\star 1} 10^{-3}$} \\
{$[1 \mathrm{~s} ; \mathrm{lr}](\mathrm{H})$} & {$[3,045 ; 3,045]^{* 10^{-3}}$} \\
$\mathrm{Lm}(\mathrm{H})$ & $124,1 * 10^{-3}$ \\
$\mathrm{~J}\left(\mathrm{~kg} \mathrm{~m}^{2}\right)$ & 0,0343 \\
\hline
\end{tabular}




\section{Drive do motor de indução trifásico}

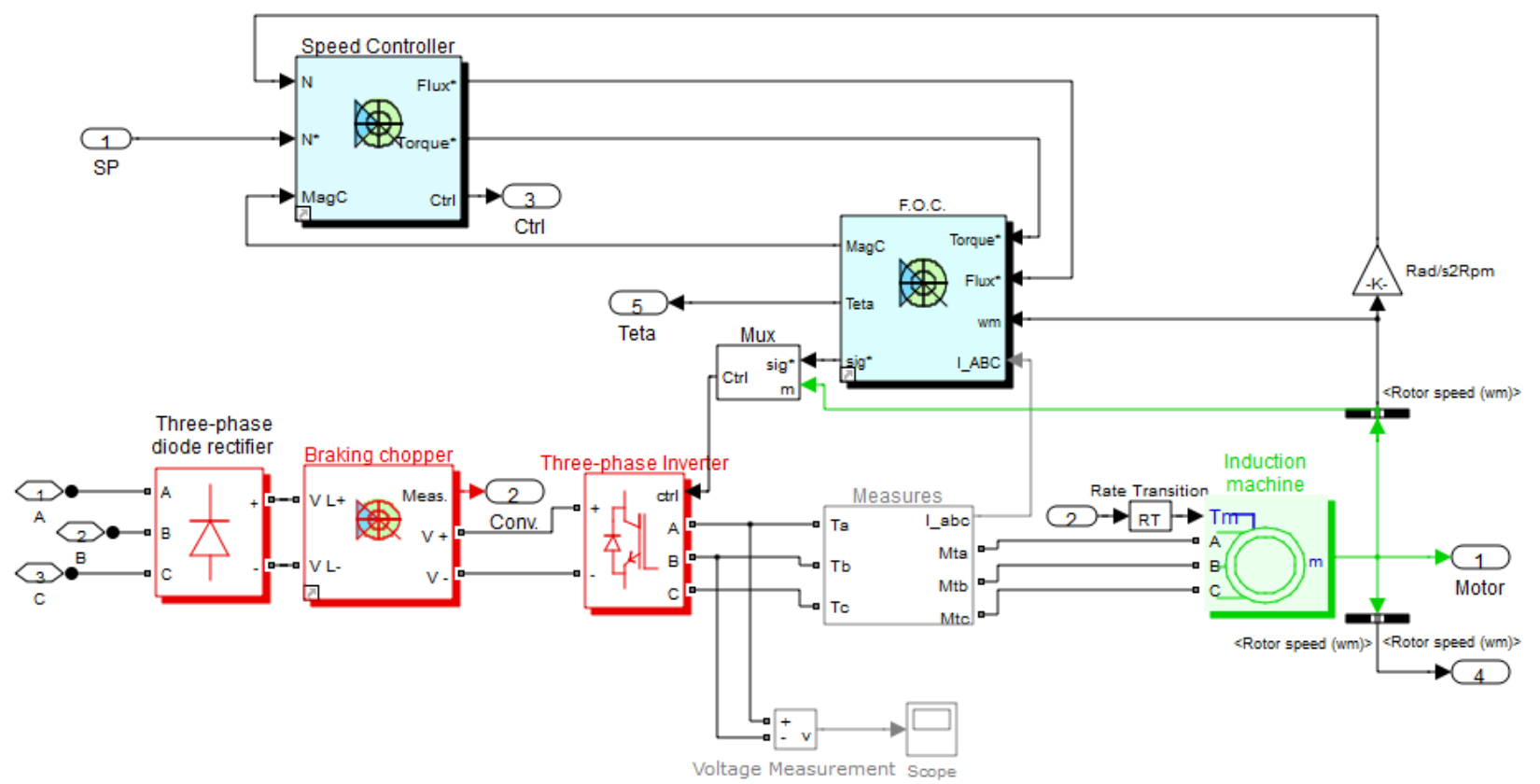

Figura 4. Estrutura da drive: conversor(vermelho), motor (verde) e controlador (azul)

Na figura 4 está representado o modelo da drive do MI3 (conversor de potência + controlador + Motor de indução) .

É visível a cadeia de transmissão de potência (conversor + motor de indução), bem como o controlador de velocidade e o controlador vetorial - bloco "F.O.C.". O valor de referência do fluxo do rotor (Flux*) é gerado pelo controlador de velocidade. Deste modo, é definida a gama de velocidades associada a fluxo constante (binário máximo constante) e a zona de enfraquecimento de campo (potência constante), de acordo com a figura 5:

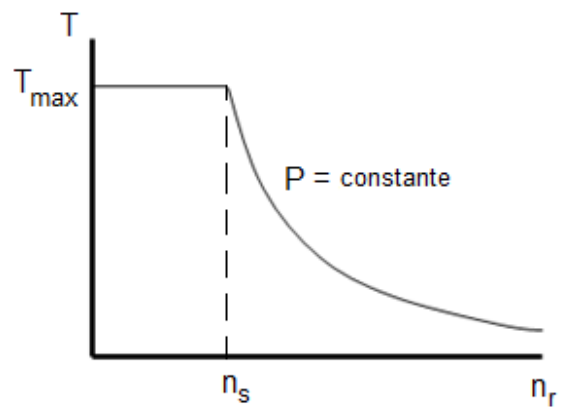

Figura 5. Zonas de funcionamento do MI: Fluxo constante (Baixas velocidades) e enfraquecimento de campo (Elevadas velocidades)
O conversor é do tipo fonte de tensão (Voltage Source Inverter - VSI), usual para a potência do motor considerado. É composto por um retificador não controlado (ponte de díodos - Three-phase diode rectifier) e um inversor (Threephase inverter) cuja tensão de saída é regulada por PWM (Pulse Width Modulation).

Existe a possibilidade de funcionamento nos 4 quadrantes, através de frenagens dissipativa (i.e., não é possível a recuperação da energia cinética do veículo): com efeito, há somente uma resistência de frenagem (bloco "Braking chopper"), onde se dá a dissipação da referida energia cinética. Com vista a evitar sobretensões no barramento DC (Vdc) devido a desacelerações bruscas ou velocidades excessivas nas descidas. A ação frenante associada à resistência é regulada através de um controlador histerético de tensão (ON se Vdc $\geq V \max$; OFF se $V d c \leq V \min$ ).

\footnotetext{
${ }^{3}$ As perdas no ferro do motor e as perdas do conversor não são incluídas.

${ }^{4}$ O símbolo * está associado à representação das grandezas de referência.
} 
Rotor FOC

A Figura 6 ilustra o conteúdo do bloco "F.O.C.", onde é implementado o algoritmo da secção 2.1.

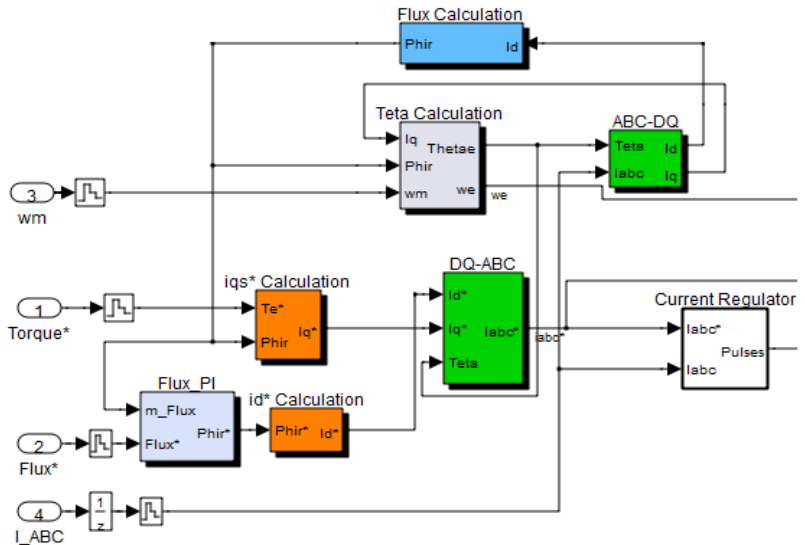

Figura 6. Implementação do Rotor FOC (indireto)

É bem visível o desacoplamento da regulação do fluxo rotórico e do binário: através do controlador PI do fluxo do rotor ("Flux_PI") é gerado o sinal Phir*, sendo calculado o valor de referência Id* através de (1).

O bloco "iqs* Calculation" determina a referência da corrente associada ao binário (Iq*), com base em (2).

Os blocos a verde estão associados à transformada de Park no referencial síncrono (ABC-DQ) e respetiva inversa (DQ$A B C)$. Neste referencial, o fluxo instantâneo do rotor é regido por (11); o seu valor é obtido através do bloco "Flux Calculation".

Finalmente, $\omega_{\mathrm{dl}}$ e $\theta_{\mathrm{f}}$ são calculados no bloco "Teta_Calculation", através de, respetivamente, (14) e (17).

O duty-cycle do trem de impulsos aplicado aos terminais das "gates" dos IGBTs do inversor é regulado em função da diferença entre labc* e labc - bloco "Current Regulator".

\section{Análise de Resultados}

Nas figuras 7-10 estão representados os resultados obtidos, para o ciclo de condução considerado (Japan: 11-Mode ).

A figura 7 ilustra as referências de velocidade (ciclo de condução) e binário (eixo motor), bem como o desempenho do MI3.
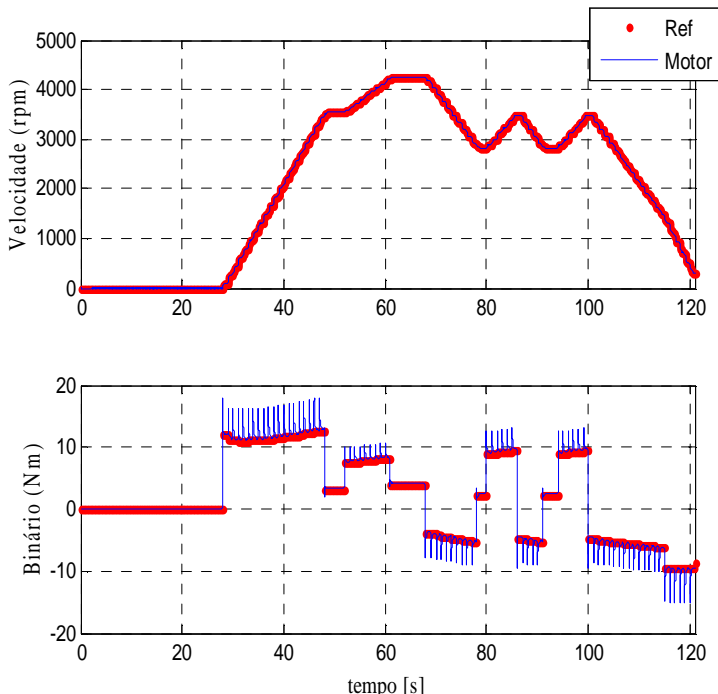

Figura 7. Perfil de velocidade e binário

A velocidade rotórica segue de modo muito fiel a referência pretendida. Naturalmente, tal resulta do facto do perfil do binário motor desenvolvido seguir a respetiva referência (modo motor: valores positivos; frenagem: valores negativos).

É de salientar o "ripple" existente nos intervalos de aceleração e desaceleração: sendo uma componente de alta frequência, o momento de inércia do sistema (motor+transmissão+carga) atenua quase na totalidade a influência desta componente, o que é visível no perfil de velocidade obtido.

As influências de Id e lq (referencial síncrono) sobre, respetivamente, o fluxo rotórico e o binário desenvolvido estão bem evidenciados na Figura 8. 

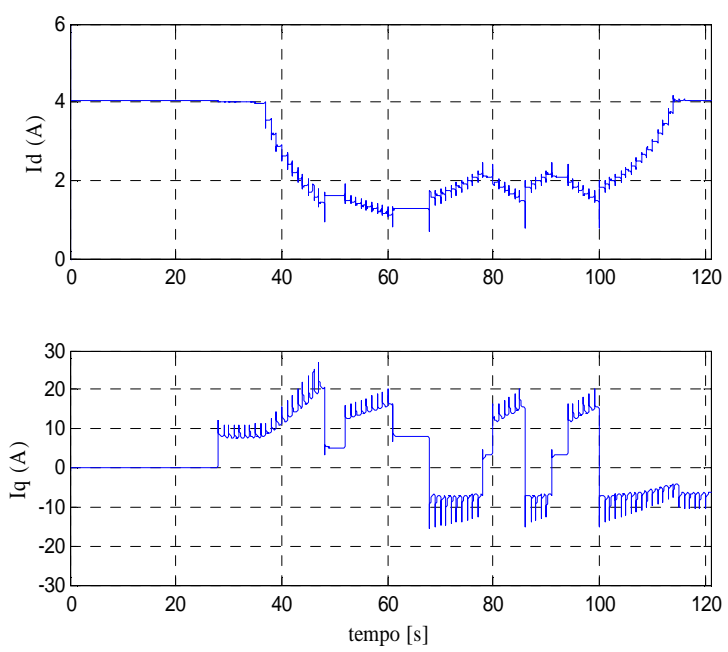

Figura 8. Perfis de $I_{d}$ e $I_{q}$ (referencial síncrono)

A fronteira entre zona de "fluxo constante" e "enfraquecimento de campo" é definida pela velocidade de sincronismo do motor (ns) - neste caso, 1500 rpm. Observase o valor constante de Id para $\mathrm{nr}<1500 \mathrm{rpm}$. Para valores superiores da velocidade (zona de enfraquecimento de campo), Id varia de forma inversamente proporcional à velocidade. Por seu turno, é visível a semelhança entre os perfis de lq e do binário desenvolvido: naturalmente, na zona de enfraquecimento de campo, lq tende a desviar-se do perfil do binário, de modo a compensar a diminuição do fluxo rotórico, de acordo com (2).

Para o ciclo de condução seleccionado, a potência instantânea é inferior à potência nominal do motor seleccionado (Figura 9).

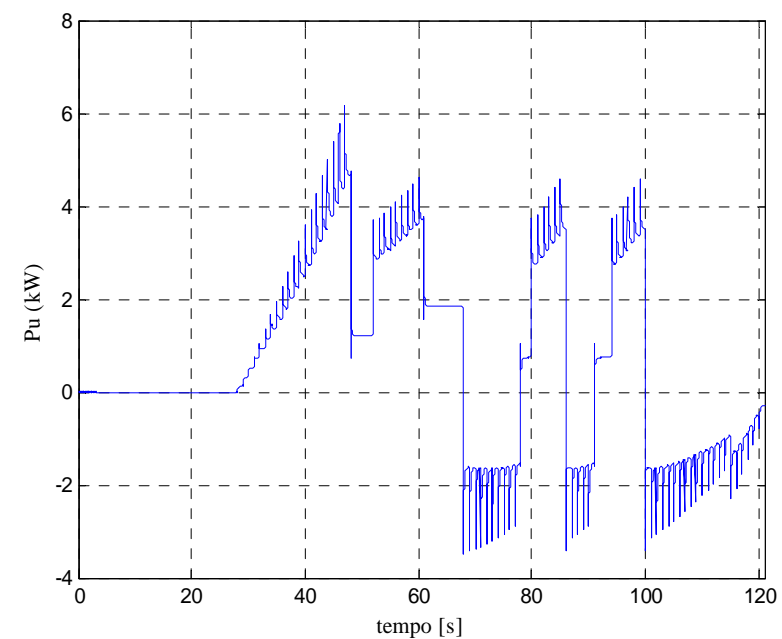

Figura 9. $P_{\text {útil }}$ do motor
No entanto, tratando-se de regimes dinâmicos, é necessária uma análise mais profunda relativamente às condições de funcionamento do motor. A título de exemplo, na Figura 10 é patente o risco de danos causados pelos efeitos térmicos, atendendo aos intervalos em que a corrente se encontra entre $20 \mathrm{~A}$ e $30 \mathrm{~A}$ (altas velocidades). Notar que o valor da corrente de pico nominal do motor é igual a: $\operatorname{sqrt}(2) * 13=18,4$ A).

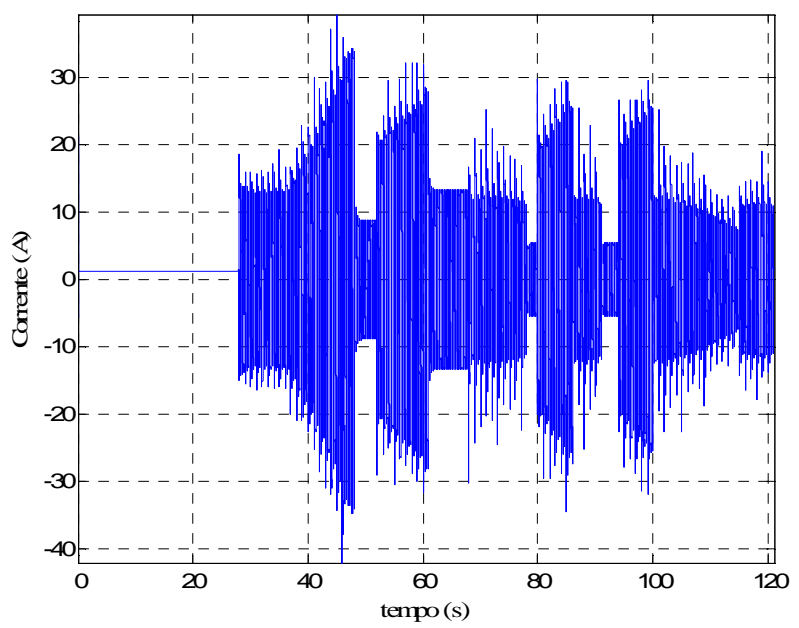

Figura 10. Corrente absorvida pelo motor

A escolha da classe de isolamento do motor e a necessidade de inclusão de ventilação forçada deverão ser devidamente ponderadas. As solicitações mecânicas nas altas velocidades (nomeadamente, nos rolamentos) é outro fator a ser analisado.

Por outro lado, o motor é submetido a uma gama de potências bastante ampla. Torna-se importante caracterizar o rendimento da máquina em múltiplos regimes de funcionamento (motor e frenagem). Os mapas de eficiência são usualmente empregues com este fim.

Na Figura 11 está representado o mapa da máquina usada (modo motor - 10 quadrante), bem como os regimes de funcionamento impostos pelo ciclo de condução escolhido (vermelho). 


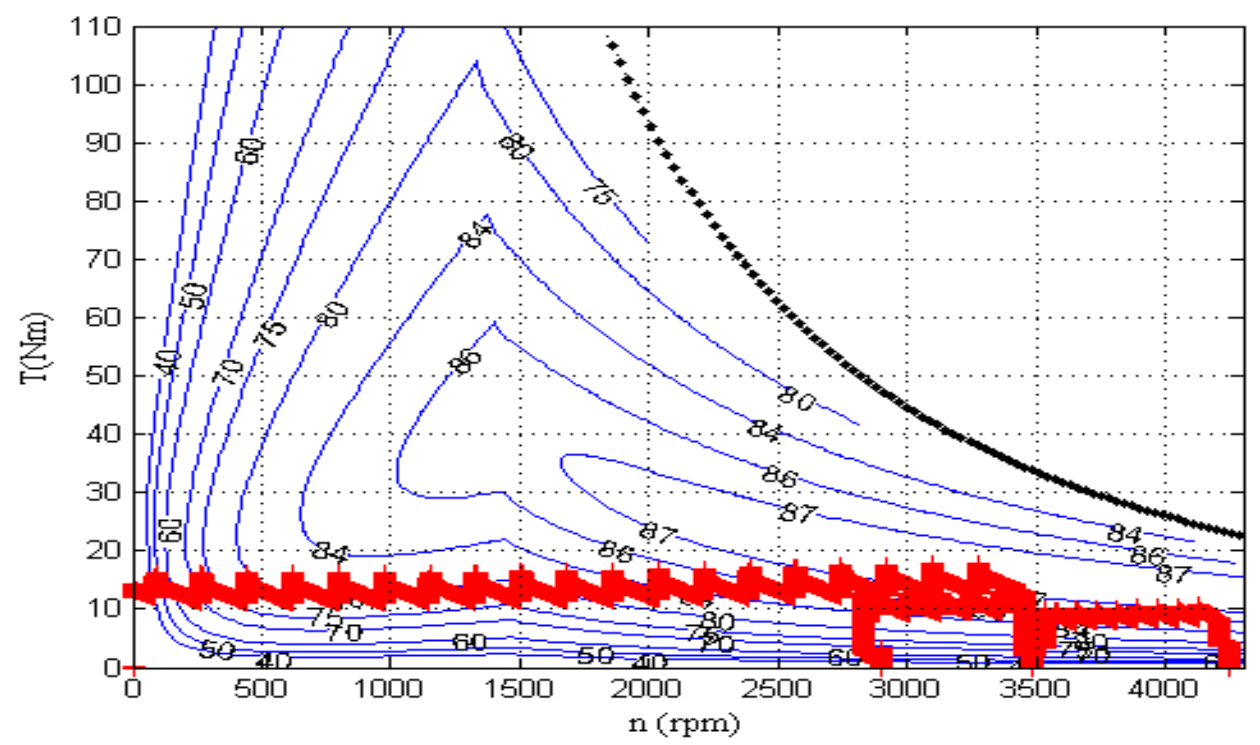

Figura 11. Mapa de eficiência do MI3 e pontos de funcionamento

Neste caso, o motor funciona com rendimentos relativamente elevados, em quase todo o ciclo de condução. As zonas de rendimentos mais baixos situam-se nas baixas velocidades, com cargas baixas. Dependendo das características dos ciclos de condução e da razão de transmissão, motor poderá funcionar preponderantemente em tais zonas. Nesses casos, com vista a melhorar o rendimento da máquina, duas opções poderão ser consideradas:

-Optar por um sistema de transmissão com múltiplas razões;

-Incluir algoritmos de otimização de fluxo, uma vez que a principal razão dos baixos rendimentos nas zonas referidas se deve ao valor demasiado elevado do fluxo magnético, face ao binário exigido [4].

\section{CONCLUSÕES}

Neste artigo procurou-se incidir nos princípios de base do controlo vetorial por orientação do campo rotórico, aplicado aos motores de indução trifásicos. Os níveis de exigência dinâmica associados aos sistemas de tração dos veículos elétricos são muito elevados (e.g., funcionamento nos 4 quadrantes, múltiplos regimes de funcionamento com variações mais ou menos bruscas, rendimentos distintos).
Como tal, a opção por um MI3 (ou outras máquinas elétricas) só é viável através de sistemas de controlo capazes de dotarem as máquinas de comportamentos dinâmicos que estejam à altura de tais exigências - o controlo vetorial (Rotor FOC) é a opção mais usual. Com base num modelo de simulação, apresentou-se um exemplo de aplicação num veículo elétrico, procurando também evidenciar algumas das condições de funcionamento da máquina elétrica, e respetivos impactos sobre a mesma. Por último, é de frisar o carácter introdutório com que se procuraram abordar estes assuntos; é essa a perspetiva com que se pretende que este artigo seja encarado.

\section{Referências}

[1] Marques, Gil (1999). “Controlo de Motores Eléctricos”, IST.

[2] Krishnan, R. (2001). Electric Motor Drives - Modeling, Analysis and Control (1 edition), Prentice Hall, ISBN 13: 978-0130910141.

[3] Guzzella, L., \& Amstutz, A. (2005), The QSS Toolbox Manual, Measurement and Control Laboratory -Swiss Federal Institute of Technology Zurich.

[4] P. Melo, R. d. Castro, and R. E. Araújo, "Evaluation of an Energy Loss-Minimization Algorithm for EVs Based on Induction Motor", Induction Motors - Modelling and Control, Intech (2012) 